\title{
Covariant entropy conjecture and concordance cosmological models
}

\author{
Song He \\ School of Physics, Peking University, Beijing, 100871, China \\ hesong@pku.edu.cn \\ Hongbao Zhang \\ Perimeter Institute for Theoretical Physics, Waterloo, Ontario, N2L 2Y5, Canada \\ Department of Astronomy, Beijing Normal University, Beijing, 100875, China \\ hzhang@perimeterinstitute.ca
}

\begin{abstract}
Recently a covariant entropy conjecture has been proposed for dynamical horizons. We apply this conjecture to concordance cosmological models, namely, those cosmological models filled with perfect fluids, in the presence of a positive cosmological constant. As a result, we find this conjecture has a severe constraint power. Not only does this conjecture rule out those cosmological models disfavored by the anthropic principle, but also it imposes an upper bound $10^{-60}$ on the cosmological constant for our own universe, which thus provides an alternative macroscopic perspective for understanding the long-standing cosmological constant problem.
\end{abstract}

KEYwORDs: Dark Matter, Dark Energy Theory, Gravity, Quantum Gravity Phenomenology. 


\section{Contents}

1. Introduction 1

2. Covariant entropy conjecture associated with cosmological dynamical horizon

3. Acceptable cosmological models constrained by covariant entropy conjecture

4. Conclusion

\section{Introduction}

The notion of dynamical horizons, free of the global and teleological deficit of event horizons, was developed quasi-locally and its properties were extensively investigated, where, in particular, the first and second laws were generalized to the black hole dynamical horizon [1, 2, 3]. Following this line further, we have recently proposed a covariant entropy bound formulation of an analogous generalized second law of thermodynamics on the black hole dynamical horizon and its generalization to cosmological dynamical horizons in FRW universes has also been conjectured [4, 5]. Moreover, with the assumption that the dominant energy condition and adiabatical evolution hold for matter, its validity has been confirmed in both Vaidya black holes and FRW universes full of matter with a fixed state of equation, no matter whether the spatial geometry is open, flat, or closed [4, 5]. All of these results suggest that dynamical horizons may also have an interpretation of thermodynamics. However, even though it turns out to be not true, as inferred above, our proposal itself can still be viewed as a covariant entropy bound conjecture on dynamical horizons and there may be some deep reasons for its validity. In fact, the conjecture is motivated partly by Bousso entropy bound conjecture [6, 7, 8, 9], and its strengthened form suggested by Flanagan, Marolf, and Wald 10]. In particular, when the dynamical horizon is spacelike, our conjecture has been proved on the basis of the strengthened form[11]. These various entropy bound conjectures, including ours, can also be interpreted as a statement of the so called holographic principle, which is believed to be manifest in an underlying quantum gravity[12, 13. 
Due to its success in many respects and justification as a possible fundamental principle, it is intriguing to know if our conjecture is able to rule out some types of cosmological models or to provide somewhat particular constraints on certain significant cosmological parameters of acceptable models, such as the cosmological constant in our own universe, which is just what we shall address in this paper. As a first step in this direction, we shall here apply our conjecture to those cosmological models with a positive cosmological constant plus the matter content satisfying the dominant energy condition, which our own universe belongs to. Remarkably, it is found that our conjecture rules out all of those cosmological models with $0<w \leq 1$ for the matter content. In addition, although our own universe satisfies our conjecture as it should, with a safety margin of 30 orders of magnitude, our conjecture alleviates the cosmological constant problem by imposing an upper bound on the cosmological constant.

Planck units are used here, i.e., $c=G=\hbar=k=1$, where $c$ is the speed of light, $G$ is the Newton constant, $\hbar$ is the Planck constant, and $k$ is the Boltzmann constant. Notation and conventions follow [14]

\section{Covariant entropy conjecture associated with cosmological dynamical horizon}

In terms of the conformal time and comoving coordinate, the FRW metric takes the form

$$
d s^{2}=a^{2}(\eta)\left[-d \eta^{2}+d \chi^{2}+f^{2}(\chi)\left(d \theta^{2}+\sin ^{2} \theta d \phi^{2}\right)\right],
$$

which describes homogeneous and isotropic universes, including, to a good degree of approximation, the portion we have seen of our own universe. Here $f(\chi)=\sinh \chi, \chi, \sin \chi$ correspond to open, flat, and closed universes, respectively.

With the line element above, the future directed null congruences orthogonal to an arbitrary sphere characterized by some value of $(\eta, \chi)$ can be chosen as

$$
k_{ \pm}^{a}=\frac{1}{2}\left[\left(\frac{\partial}{\partial \eta}\right)^{a} \pm\left(\frac{\partial}{\partial \chi}\right)^{a}\right]
$$

whose expansions are given by 15

$$
\theta_{ \pm}=\frac{\dot{a}}{a} \pm \frac{f^{\prime}}{f}
$$

where the $\operatorname{dot}($ prime) denotes differentiation with respect to $\eta(\chi)$, and the sign $+(-)$ represents the null congruence directed at larger(smaller) values of $\chi$. Note that the

second term is given by $\operatorname{coth} \chi, \frac{1}{\chi}, \cot \chi$ for open, flat, and closed universes, respectively. 
In particular, this term diverges when $\chi \rightarrow 0$, and it also diverges when $\chi \rightarrow \pi$ for a closed universe.

Then the cosmological dynamical horizon can be defined geometrically as a threedimensional hypersurface foliated by those spheres at which at least there exists one orthogonal null congruence with vanishing expansion. Thus the cosmological dynamical horizon $\chi_{c}(\eta)$ is identified by solving the equation

$$
h= \pm\left.\frac{f^{\prime}}{f}\right|_{\chi=\chi_{c}}
$$

where $h \equiv \frac{\dot{a}}{a}$. There is one solution for open and flat universes, while for a closed universe, there are generally two solutions, which are symmetrically related to each other by $\chi_{c}^{2}(\eta)=\pi-\chi_{c}^{1}(\eta)$. Now the covariant entropy conjecture on cosmological dynamical horizon can be addressed as follows [4]: Let $A(\eta)$ be the area of the cosmological dynamical horizon at the conformal time $\eta$, then the entropy flux $S$ through the cosmological dynamical horizon between the conformal times $\eta$ and $\eta^{\prime}$ must satisfy $S \leq \frac{\left|A(\eta)-A\left(\eta^{\prime}\right)\right|}{4}$ if the dominant energy condition holds for matter.

It is noteworthy that unlike for the black hole dynamical horizon, we have no restriction on the signature of cosmological dynamical horizons in our definition, which means our cosmological dynamical horizons can be spacelike, null, or timelike 15. This extension, however, does not ruin the validity of our covariant entropy conjecture in the cosmological context. As demonstrated in [四], our conjecture always holds regardless of the signature of cosmological dynamical horizons.

\section{Acceptable cosmological models constrained by covariant en- tropy conjecture}

If as usual the matter content of FRW universes is assumed to be described by the perfect fluid with energy momentum tensor

$$
\begin{aligned}
T_{a b}= & a^{2}(\eta)\left\{\rho(\eta)(d \eta)_{a}(d \eta)_{b}+p(\eta)\left[(d \chi)_{a} d(\chi)_{b}\right.\right. \\
& \left.\left.+f^{2}(\chi)\left((d \theta)_{a}(d \theta)_{b}+\sin ^{2} \theta(d \phi)_{a}(d \phi)_{b}\right)\right]\right\}
\end{aligned}
$$

then by the Einstein equation with a positive cosmological constant $\Lambda$, we have

$$
\begin{aligned}
3\left(h^{2}+K\right) & =8 \pi \rho a^{2}+\Lambda a^{2}, \\
-\left(h^{2}+2 \dot{h}+K\right) & =8 \pi p a^{2}-\Lambda a^{2},
\end{aligned}
$$


where $K=1,0,-1$ correspond to open, flat, and closed universes, respectively. From here, we can further obtain

$$
\begin{aligned}
\dot{h} & =-\frac{4 \pi}{3}[(1+3 w) \rho-\lambda] a^{2}, \\
h^{2}+K-\dot{h} & =4 \pi(1+w) \rho a^{2},
\end{aligned}
$$

and the energy momentum conservation equation

$$
\dot{\rho}=-3(1+w) \rho h
$$

where $-1 \leq w \equiv \frac{p}{\rho} \leq 1$ due to the dominant energy condition, and $\lambda \equiv \frac{\Lambda}{4 \pi}$.

To proceed, we further assume that the evolution of FRW universes is adiabatical, which implies the conservation of the entropy current associated with the matter, i.e., $\nabla_{a} s^{a}=0$. Hence the entropy current can be formulated as

$$
s^{a}=\frac{s}{a^{4}}\left(\frac{\partial}{\partial \eta}\right)^{a},
$$

where $s$ is actually the ordinary comoving entropy density, constant in space and time.

On the other hand, according to eq.(2.4), we have

$$
\dot{\chi}_{c}=\mp \frac{\dot{h}}{h^{2}+K} .
$$

Note that at any moment the area of the cosmological dynamical horizon is give by $A=4 \pi a^{2} f^{2}\left(\chi_{c}\right)$. Accordingly we can work out its time derivative, i.e.,

$$
\begin{aligned}
\dot{A} & =8 \pi a^{2} f^{2}\left(\chi_{c}\right)\left(h+\frac{\dot{f}\left(\chi_{c}\right)}{f\left(\chi_{c}\right)}\right)=8 \pi a^{2} f^{2}\left(\chi_{c}\right)\left(h+\frac{f^{\prime}\left(\chi_{c}\right) \dot{\chi}_{c}}{f\left(\chi_{c}\right)}\right) \\
& =8 \pi a^{2} f^{2}\left(\chi_{c}\right) \frac{h}{h^{2}+K}\left(h^{2}+K-\dot{h}\right) .
\end{aligned}
$$

Obviously, by eq.(3.2) and eq.(3.5), the increase or decrease of area with time only depends on whether the universe is expanding or contracting. In what follows we shall only focus on the expanding universes, i.e., $h \geq 0$, where the corresponding area monotonically increases with the evolution of time.

We shall now check whether our conjecture is satisfied for those universes mentioned above. However, as demonstrated in figure 1, it is noteworthy that there is an obvious difference between $\dot{\chi}_{c} \leq 0$ and $\dot{\chi}_{c} \geq 0$. Thus employing the conservation of the entropy current and Gauss theorem, our conjecture can be equivalently expressed as

$$
\frac{\dot{A}}{4}+\dot{S} \geq 0
$$




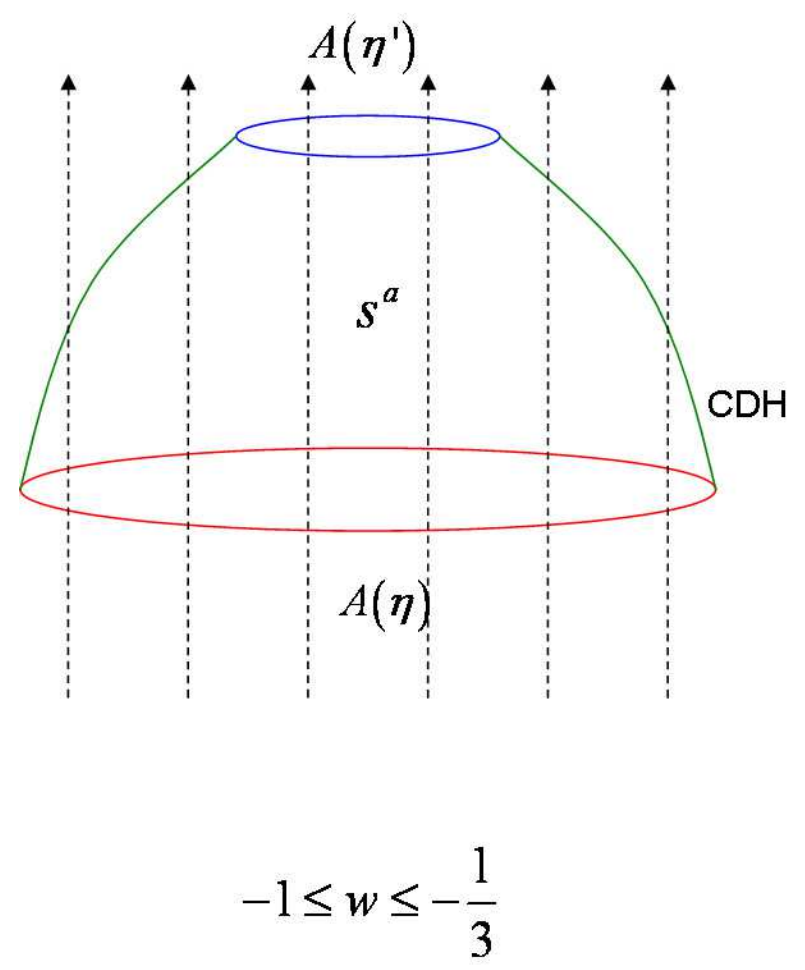

(a)

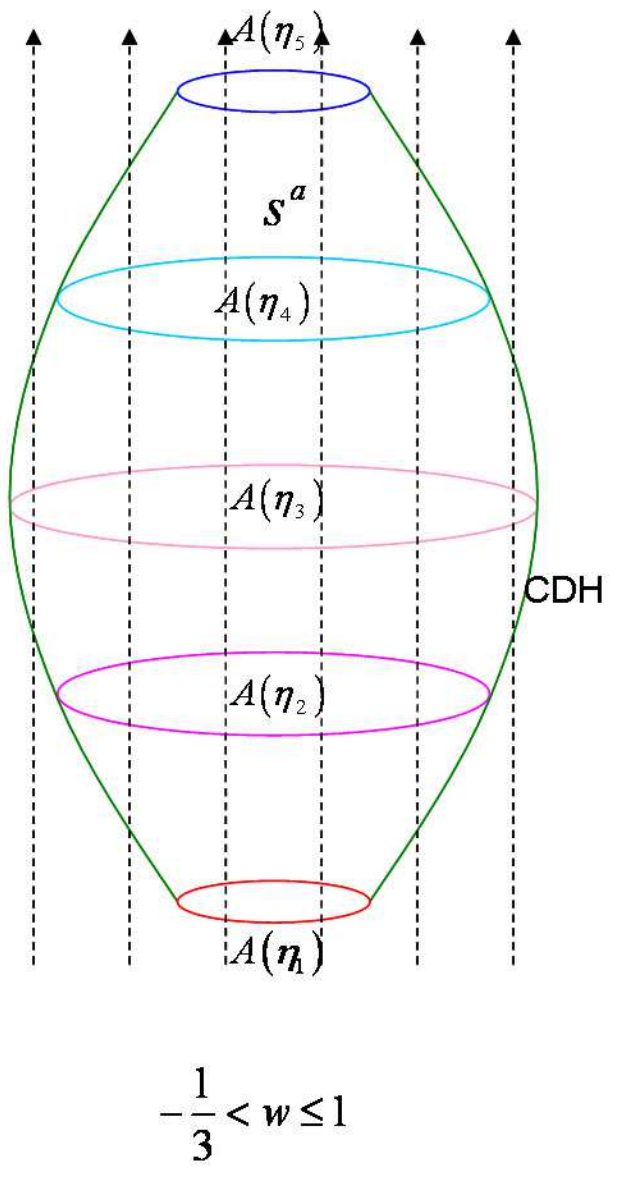

(b)

Figure 1: The cosmological dynamical horizon with the entropy current flowing through it in the conformal coordinate. When $\dot{\chi}_{c} \leq 0$, the entropy current flows across the cosmological dynamical horizon from the interior region to the exterior one, while it flows from the exterior region to the interior one for $\dot{\chi}_{c} \geq 0$.

for $\dot{\chi}_{c} \leq 0(\dot{h} \geq 0)$, and

$$
\frac{\dot{A}}{4}-\dot{S} \geq 0
$$

for $\dot{\chi}_{c} \geq 0(\dot{h} \leq 0)^{1}$. Here $S$ denotes the entropy flux through the interior region $\chi \leq \chi_{c}$,

${ }^{1}$ As explained in 叫, this can be reasonably taken as the generalized second law of thermodynamics in the cosmological context. 
given by

$$
S=4 \pi s \int_{0}^{\chi_{c}} d \chi f^{2}(\chi)
$$

whereby we have

$$
\dot{S}=4 \pi s f^{2}\left(\chi_{c}\right) \dot{\chi}_{c}=-4 \pi s f^{2}\left(\chi_{c}\right) \frac{\dot{h}}{h^{2}+K} .
$$

So for $-1 \leq w \leq-\frac{1}{3}$, our conjecture requires

$$
s \leq \frac{\sqrt{3\left(8 \pi \rho a^{2}+\Lambda a^{2}-3 K\right)}(1+w) \rho a^{2}}{2[\lambda-(1+3 w) \rho]},
$$

where eq.(3.2), eq.(3.4), and eq.(3.5) have been used. Furthermore, by eq.(3.6), we know the energy density $\rho \propto a^{-3(1+w)}$. Thus the rhs of the above inequality is an increasing function of $a$, which is similar to our previous result without the cosmological constant [4]. That is to say, the bound will hold forever once it holds at some early time, where classical general relativity becomes an effective theory for successfully describing the evolution of universes. This is expected since the cosmological constant corresponds to $w=-1$ which falls into the branch $-1 \leq w \leq-\frac{1}{3}$ and a combination of the cosmological constant with the matter content in this branch will not change the situation to violate our conjecture.

Similarly, for the case of $-\frac{1}{3}<w \leq 1$, our conjecture is equivalent to

$$
s \leq \frac{\sqrt{3\left(8 \pi \rho a^{2}+\Lambda a^{2}-3 K\right)}(1+w) \rho a^{2}}{2[(1+3 w) \rho-\lambda]}
$$

when $(1+3 w) \rho>\lambda$, and

$$
s \leq \frac{\sqrt{3\left(8 \pi \rho a^{2}+\Lambda a^{2}-3 K\right)}(1+w) \rho a^{2}}{2[\lambda-(1+3 w) \rho]}
$$

when $(1+3 w) \rho<\lambda$, which apparently corresponds to later stages of expanding universes. For simplicity but without loss of generalization, we shall restrain our later discussions to the flat case, i.e., $K=0$. Then after a straightforward calculation, we find that the rhs of inequality (3.15) is an increasing function of $a$. However, the rhs of inequality (3.16) is a decreasing function of $a$ for $0 \leq w \leq 1$; on the other hand, for $-\frac{1}{3}<w<0$, it decreases with $a$ in the region of $\lambda<\frac{1+\frac{2}{\sqrt{1+3 w}}}{1-\sqrt{1+3 w}}(1+3 w) \rho$, and then increases with $a$ in the region of $\lambda>\frac{1+\frac{2}{\sqrt{1+3 w}}}{1-\sqrt{1+3 w}}(1+3 w) \rho$. Obviously, here we see a very different situation from our previous one. Namely, for $-\frac{1}{3}<w \leq 1$, the bound may not be guaranteed to hold at some late stages even if it holds at reasonable early 
stages. Speaking specifically, for $-\frac{1}{3}<w \leq 0$, whether the bound will hold depends critically on whether the inequality (3.16) will hold at the point where the minimum for the rhs occurs. What is worse, for $0<w \leq 1$, the bound is doomed to be violated at late stages of the evolution of expanding universes since the rhs approaches zero as $a \rightarrow \infty$ by $\rho \propto a^{-3(1+w)}$. However, one may regard this observation as an indication that some types of cosmological models can be ruled out by our conjecture. Putting this another way, if we take this viewpoint seriously, we can claim that our conjecture rules out those cosmological models with a mixed content of a positive cosmological constant and matter satisfying a fixed equation of state of $0<w \leq 1$.

Although all the discussion above is confined to only one kind of matter content besides a positive cosmological constant, it is easy to generalize to a more realistic cosmological model where various types of matter are combined with a positive cosmological constant. In particular, for the currently favored concordance $\Lambda$ CDM model, where $\rho=\frac{\rho_{r}^{0} a_{0}^{4}}{a^{4}}+\frac{\rho_{m}^{0} a_{0}^{3}}{a^{3}}$, on the basis of eq.(3.16) and taking into account that the dust has been the dominant matter content, our conjecture requires

$$
s \sqrt{\Lambda} \leq 2 \sqrt{3} \pi \rho_{m}^{0}
$$

where $\rho_{m}^{0}$ denotes the energy density of dust today, and the present scale factor is set to be $a_{0}=1$, which implies that $s$ represents the entropy density today. This is a remarkable result because it establishes a novel relation governing the cosmological constant, matter entropy density and dust energy density. For our own universe, as is well known, $\Lambda \sim 10^{-120}$ and $\rho_{m}^{0} \sim \frac{1}{3} \Lambda$, so from our conjecture it follows that the present entropy density should be less than $10^{-60}$, which is satisfied with a wide safety margin, since the realistic entropy density is around of order $10^{-90}$ today. That is to say, our conjecture supports the existence of our own universe as it should do. On the other hand, if we take the present matter entropy density and dust energy density as input data, our conjecture gives an upper bound on the cosmological constant, i.e., $\Lambda<10^{-60}$, which obviously alleviates the cosmological constant problem of the cosmological constant being so small in Planck units. Last but not least, the presence of cosmological constant, albeit small, appears to be in favor of a bio-friendly universe: to have our conjecture satisfied, there should be dust matter in our universe, which is assumed to be a very basic condition for the creation of life since it is the dust matter that constitutes galaxies, stars, planets, and creatures including human beings.

\section{Conclusion}

Without knowledge of its microscopic makeup and specific dynamics, the use of general principles to investigate a system can be very rewarding. Examples of such principles 
include the widely accepted thermodynamics and recently recognized holographic principle. The holographic principle, albeit confirmed in limited concrete models, is believed to be a law of physics that captures one of the most crucial aspects of quantum gravity, and thus a key insight for guiding the progressing search for a successful unified theory.

As a compelling pattern for the holographic principle, and an equivalent formulation of generalized second law of thermodynamics as well, our recently proposed covariant entropy conjecture has been applied to cosmological models with the presence of a positive cosmological constant. As a result, it is shown that our conjecture places a severe constraint on acceptable cosmological models. Not only does this conjecture rule out those cosmological models disfavored by the anthropic principle, but also it imposes an upper bound $10^{-60}$ on the cosmological constant for our own universe, which thus opens an alternative macroscopic perspective to shed light on the long-standing cosmological constant problem. Although this upper bound does not fix the exceedingly tiny value of cosmological constant, it is plausible that there are other contributions to entropy density which we have not taken into account, and a more complete picture of constituents and structures of the universe, will yield a better estimate of the entropy density and finally resolve the cosmological constant problem.

We conclude with an honest caveat. Although the results obtained so far are particularly attractive as well as consistent with our observational data, there remains a possibility that our starting conjecture proves incorrect. It may be quite successful in many respects only as a coincidence, but one should regard it as as a warning, showing that our covariant entropy conjecture may require additional justification and reformulation where it is violated, rather than being a criterion for singling out acceptable models. Therefore not only is it important to provide more indirect or peripheral justifications, but also it is needed to signify a deeper origin of our conjecture in an underlying quantum theory of gravity, such as causal set theory, loop quantum gravity, or string theory 16, 17, 18, 19.

\section{Acknowledgements}

We are deeply indebted to Niayesh Afshordi, Achim Kempf, Federico Piazza, Maxim Pospelov, Lee Smolin, Rafael Sorkin, and Leonard Susskind for their helpful suggestions and comments. HZ would like to express much gratitude to Stephen Hawking for his vivid communication on the anthropic principle and related issues. He is also grateful to Xiao Liu for valuable criticisms leading to improvement. The work by SH was supported by NSFC(nos.10235040 and 10421003). HZ was supported in part by the Government of China through CSC(no.2007102530). This research was supported by Perimeter Institute for Theoretical Physics. Research at Perimeter 
Institute is supported by the Government of Canada through IC and by the Province of Ontario through MRI.

\section{References}

[1] A. Ashtekar and B. Krishnan, Phys. Rev. Lett. 89, 261101(2002)[arXiv:gr-qc/0207080].

[2] A. Ashtekar and B. Krishnan, Phys. Rev. D 68, 104030(2003)[arXiv:gr-qc/0308033].

[3] A. Ashtekar and B. Krishnan, Living Rev. Rel. 7, 10(2004)[arXiv:gr-qc/0407042].

[4] S. He and H. Zhang, JHEP 10, 077(2007)[arXiv:0708.3670].

[5] S. He and H. Zhang, JHEP 12, 052(2007)[arXiv:0712.1313].

[6] R. Bousso, JHEP 07, 004(1999)[arXiv:hep-th/9905177].

[7] R. Bousso, JHEP 06, 028(1999)[arXiv:hep-th/9906022].

[8] R. Bousso, Class. Quant. Grav. 17, 997(2000)[arXiv:hep-th/9911002].

[9] R. Bousso, Rev. Mod. Phys. 74, 825(2002)[arXiv:hep-th/0203101].

[10] E. E. Flanagan, D. Marolf, and R. M. Wald, Phys. Rev. D 62, 084035(2000)[arXiv:hep-th/9908070].

[11] S. Gao and X. Wu, JHEP 08, 005(2008)[arXiv:0807.4574].

[12] G. 't Hooft, arXiv:gr-qc/9310026.

[13] L. Susskind, J. Math. Phys. 36, 6377(1995)[arXiv:hep-th/9409089].

[14] R. M. Wald, General Relativity(University of Chicago Press, Chicago, 1984).

[15] I. Ben-Dov, Phys. Rev. D 70, 124031(2004)[arXiv:gr-qc/0408066].

[16] D. Dou and R. D. Sorkin, Found. Phys. 33, 279(2003)[gr-qc/0302009].

[17] D. Rideout and S. Zohren, Class. Quant. Grav. 23, 6195(2006)[gr-qc/0606065].

[18] A. Ashtekar, J. Baez, A. Corichi and K. Krasnov, Phys. Rev. Lett. 80, 904(1998)[gr-qc/9710007].

[19] A. Strominger and C. Vafa, Phys. Lett. B 379, 99(1996)[hep-th/9601029]. 